\title{
Análisis de costo-utilidad del uso de detemir en pacientes con diabetes tipo $2^{*}$
}

\section{Cost-Utility Analysis of the Use of Detemir in Patients with Type 2 Diabetes}

\section{Análise custo-efetividade para o uso do detemir em pacientes com Diabetes tipo 2}

Fecha de recepción: 04-02-15 Fecha de aceptación: 11-06-15 Disponible en línea: 30-06-15 doi: 10.11144/Javeriana.rgyps18-28.acuu

Como citar este artículo:

Romero-Prada ME, Huérfano-Rey LM, Luz-Páez M, Acero-Acero G, Alfonso-Quiñones PA. Análisis de costo-utilidad del uso de detemir en pacientes con diabetes tipo 2. Rev. Gerenc. Polít. Salud. 2015; 14(28): 190-201. http://dx.doi.org/10.11144/Javeriana.rgyps18-28.acuu

\author{
Martín Emilio Romero-Prada** \\ Lina María Huérfano-Rey*** \\ Mary Luz Páez**** \\ Germán Acero-Acero $* * * * *$ \\ Pamela Andrea Alfonso-Quiñones******
}

\footnotetext{
Artículo de investigación. Nombre del proyecto: Análisis de costo-utilidad del uso de detemir en pacientes con diabetes tipo 2. Entidad financiadora: Novo Nordisk Colombia. Proyecto desarrollado desde septiembre del 2014 hasta enero del 2015. Por favor remitir la correspondencia a Martín Emilio Romero Prada, Carrera 71B \# 116A-12, Bogotá, Colombia.

** Candidato a doctor en Salud Pública de la Universidad Nacional de Colombia. Codirector de la Fundación Salutia - Centro de Investigaciones en Economía, Gestión y Tecnologías en Salud. Correo electrónico: martin.romero@ salutia.org.

*** Máster en Salud Pública de la Pontificia Universidad Javeriana. Coordinadora clínica de la Fundación Salutia - Centro de investigaciones en Economía, Gestión y Tecnologías en Salud, Bogotá, Colombia. Correo electrónico: lina.huerfano@ salutia.org.

***** Especialista en Epidemiología de la Universidad del Rosario y bacterióloga de la Pontificia Universidad Javeriana. Investigadora de la Fundación Salutia - Centro de Investigaciones en Economía, Gestión y Tecnologías en Salud, Bogotá, Colombia. Correo electrónico: maryluz.paez@salutia.org.

****** Profesional en Finanzas y Negocios Internacionales de la Fundación Universitaria Monserrate. Investigador de la Fundación Salutia - Centro de Investigaciones en Economía, Gestión y Tecnologías en Salud, Bogotá, Colombia. Correo electrónico: german.acero@salutia.org.

****** Médico con Énfasis en Salud Pública de la Universidad de La Sabana. Investigadora de la Fundación Salutia - Centro de Investigaciones en Economía, Gestión y Tecnologías en Salud, Bogotá, Colombia. Correo electrónico: pamela. alfonso@salutia.org.
} 


\section{Resumen}

Objetivo: realizar un análisis de costo-utilidad para evaluar el tratamiento con las diferentes opciones de insulina en pacientes diabéticos tipo 2. Metodología: partiendo del análisis realizado previamente por Romero et al., se actualizó la información y se incluyó un análisis específico para calidad de vida. El modelo de Markov simuló pacientes tratados con insulina NPH, detemir o glargina. Se consideraron los eventos de hipoglucemia más sus complicaciones. Los costos corresponden al precio máximo de regulación por dosis. Los costos de eventos son tomados de registros del sistema de información de transacciones de entidades prestadoras de salud al año 2014. Resultados: los AVAC con detemir se calculan en 2,604, en comparación con 2,545 con glargina y 2,533 con NPH. Los análisis de sensibilidad muestran dominancia de detemir. Conclusiones: detemir es una opción favorable por su seguridad, efectividad y costo, demostrando beneficios medidos en AVAC sobre sus comparadores.

Palabras clave: diabetes mellitus tipo 2; insulina; calidad de vida; análisis de costo-utilidad; efectividad

\section{Abstract}

Objective: Performing a cost-utility analysis to assess the treatment with different insulin options in patients with Type 2 Diabetes. Methodology: Based on the analysis previously performed by Romero et al., we updated the information and included an specific analysis for the quality of life. The Markov model simulated patients treated with NPH, determir, or glargine insulin. We considered the hypoglycemia events and their complications. The costs corresponded to the maximum regulated price per dose. The event costs were taken from the transaction information system records of health care providers in 2014. Results: The QALY with detemir are calculated as 2.604 , compared to 2.545 with glargine, and 2.533 con NPH. The sensitivity analysis show determir dominance. Conclusions: Detemir is a favorable option due to its safety, effectiveness, and cost, with proven benefits measured in QALY over the other items being compared.

Keywords: diabetes mellitus type 2; insulin; quality of life; cost-utility analysis; effectiveness

\section{Resumo}

Objetivo: realizar análise de custo-efetividade para avaliar o tratamento com as diferentes opções de insulina em pacientes diabéticos tipo 2. Metodologia: partindo da análise realizada previamente por Romero et al., foi atualizada a informação e incluiu-se análise específica para qualidade de vida. O modelo de Markov simulou pacientes tratados com insulina NPH, detemir ou glargina. Eventos de hipoglicemia foram considerados além das complicações. Os custos correspondem ao preço máximo de regulamentação por dose. Os custos de eventos são tirados de registos do sistema de informação de transações de entidades prestadoras de saúde no ano 2014. Resultados: os AVAC do detemir calculam-se em 2,604, em comparação com 2,545 da glargina e 2,533 do NPH. As análises de sensibilidade demostram dominância de detemir. Conclusões: detemir é uma opção favorável por sua segurança, efetividade e custo, demostrando benefícios medidos em AVAC sobre os comparadores.

Palavras-chave: diabetes mellitus tipo 2; insulina; qualidade de vida; análise de custo-efetividade; efetividade 


\section{Introducción}

La diabetes es una enfermedad crónica que se caracteriza por un aumento en la glucemia que puede ser secundario a una deficiencia en la producción de la insulina o la ineficacia en la utilización de esta hormona por los diferentes tejidos del cuerpo (1). Tiene un origen multifactorial, por lo cual requiere un manejo sobre cada uno de los factores que se encuentren relacionados con su aparición (2).

En la actualidad, el número aproximado de diabéticos en el mundo es de 387 millones de personas, con una prevalencia de 8,3\% y una edad promedio entre los 40 y los 59 años de edad. De ese total, $77 \%$ pertenece a países de ingresos medios y bajos, los cuales han realizado un gasto anual de 612000 millones de dólares para el manejo de la enfermedad. Se espera que para el año 2035 haya un incremento de 205 millones de diabéticos. Esto lleva a que esta enfermedad sea estudiada, con el fin de elaborar elementos que ayuden a su minimización, progresión y complicación (3). En el 2014, en el contexto latinoamericano, Colombia reportó 2,1 millones de personas con diabetes y en la actualidad tiene una prevalencia de $7,17 \%$ para la población comprendida entre los 20 y los 79 años de edad (3).

Teniendo en cuenta el impacto de la diabetes sobre la población y la complejidad de su tratamiento, es necesario estudiar los beneficios de las diferentes tecnologías disponibles para el control de los niveles de glucemia, y así comprender cómo estas pueden disminuir el riesgo de complicaciones o muerte por esta enfermedad.

Las recomendaciones de salud pública para el control de la enfermedad se enfocan en la prevención primaria y secundaria, pues, además de ir en aumento, esta enfermedad se asocia con complicaciones macro y microvasculares que aumentan los gastos en salud (3). Entre las recomendaciones de tratamiento clínico-farmacológico se encuentra el inicio de la terapia con metformina, con un control médico y paraclínico a los tres meses de iniciado el tratamiento. En los pacientes que no se logra cumplir con la meta de control glucémico, se inicia terapia con una segunda molécula, cuyo mecanismo de acción es diferente, no sin antes tener en cuenta las comorbilidades que esta pueda generarle al paciente (2).

Dentro de las posibilidades de tratamiento se encuentran las insulinas. De estas, la insulina NPH se caracteriza por el alto riesgo de producir hipoglucemias (4), comparada con detemir y glargina. En el estudio de Singh et al. se comparó la insulina detemir, que es una insulina análoga de acción prolongada, cuyo objetivo es suplir la secreción basal nocturna de insulina entre comidas, para el control de la producción de la glucosa en el hígado durante el periodo interprandial (5), en comparación con la insulina NHP, insulina de acción intermedia que ha demostrado tener periodos de disminución de su efecto y una frecuencia mayor de hipoglucemias. En este mismo estudio se encontró que existe una reducción significativa en el riesgo de presentación de hipoglucemias graves con detemir, si se compara con la insulina NPH de RR 0,74 IC 95\% (0,58-0,96) (6). De igual forma, el estudio de Pieber et al. reveló que detemir disminuye el riesgo de hipoglucemia grave, en comparación con la insulina glargina, con un RR 0,25 IC 95\% (0,07-0,86) (7).

En un estudio prospectivo no intervencionista multicéntrico, realizado por Zilova et al., en el que fueron analizados datos de 15545 personas que iniciaron el tratamiento con insulina detemir, se pudo evidenciar la disminución en la presentación de casos de hipoglucemia grave. También se reportó como resultado una disminución en el pro- 
medio del peso corporal de los pacientes, en la presión arterial y el perfil lipídico. Además, también fue analizada la calidad de vida y se concluyó que la medida mejora con el uso de detemir (8).

Teniendo en cuenta que la diabetes es una enfermedad que causa un impacto en la calidad de vida de los pacientes, y que varios estudios clínicos han demostrado efectividad y seguridad de detemir (9-11), así como costo-efectividad, de acuerdo con el estudio realizado por Romero et al. (12), en el que se encontraron beneficios en su uso a bajo costo, se vio la necesidad de realizar esta evaluación de costo-utilidad del uso de las insulinas detemir, glargina y NPH, teniendo en cuenta los cambios en la calidad de vida de los pacientes con diagnóstico de diabetes mellitus tipo 2 (DM2), aplicando las condiciones de regulación de precios existentes en el país.

Por tanto, este estudio busca evaluar los beneficios en términos de calidad de vida del paciente que sufre de DM2 en Colombia y que en su tratamiento utiliza alguna de estas insulinas, reconociendo que el control intensivo de la glucemia permite retrasar y prevenir las complicaciones que están asociadas a esta enfermedad, además de establecer que la presentación de hipoglucemias muestra una relación con el desarrollo de eventos macro y microvasculares y/o muerte, según lo señalado en el estudio realizado por Zoungas et al. (13).

\section{Metodología}

Esta evaluación partió de un estudio económico previamente realizado por Romero et al. (12), en el que se evaluó la costo-efectividad de detemir, en comparación con la insulina NPH y la glargina, pero sin el ajuste a los precios que fue realizado por el Ministerio de Salud y Protección Social en el 2014, luego de la publicación del estudio.
Se desarrolló una evaluación económica tipo costo-utilidad, con el propósito de comparar el uso de detemir, la glargina y la insulina $\mathrm{NPH}$, para lo cual se tomó como desenlace de análisis los años de vida ajustados por calidad (AVAC), en un horizonte temporal de cinco años. Además, se ajustó el modelo de cadenas de Markov probabilístico desarrollado por Romero et al. (12) y se incluyó en el análisis el desenlace de calidad de vida como una medida de utilidad para cada estado de salud, el cual se presenta en el modelo. Las probabilidades fueron tomadas de estudios reportados en la literatura hasta el año 2014, actualizando la información tenida en cuenta en el estudio anterior, relacionada con efectividad y seguridad de cada una de las moléculas en evaluación (12).

Debido a que en Colombia no se cuenta con una medida de calidad de vida para los pacientes con diagnóstico de DM2, dentro de la revisión fue necesario buscar evidencia que reportara medidas de utilidad para los diferentes estados tenidos en cuenta dentro del modelo, como lo son: utilidad del paciente que sufre eventos microvasculares, utilidad del paciente que sufre eventos macrovasculares y utilidad de los pacientes que presentan hipoglucemias.

Esta revisión se realizó con una ventana de búsqueda de diez años y se estableció como requisito que los estudios reportaran medidas de utilidad elaborados a partir del EQ-5D, como los sugiere el Manual metodológico para la elaboración de evaluaciones económicas en salud del Instituto de Evaluación Tecnológica en Salud (IETS) de Colombia (14).

\section{Diseño del modelo}

En el modelo de cadenas de Markov probabilístico, el paciente que recibe como tratamiento insulina (NPH, detemir o glargina) 
puede presentar o no episodios de hipoglucemia durante cada ciclo y puede desarrollar eventos macrovasculares, microvasculares y/o morir. El modelo tiene en cuenta si en los últimos cinco años el paciente desarrolló al menos un evento de hipoglucemia, generando una diferencia en el riesgo comparada con aquellos pacientes que no han presentado eventos hipoglucémicos (figura 1).

Teniendo en cuenta que la presentación de eventos de hipoglucemia aparece registrada como una probabilidad dentro de una población, se simuló una cohorte hipotética de 10000 pacientes para cada uno de los tres brazos del modelo. La probabilidad de presentar episodios o eventos se mueve en el intervalo de confianza reportado por los estudios clínicos, en un horizonte temporal de cinco años y con ciclos de veintiséis semanas.

En la tabla 1 se presentan las principales fuentes de entrada de probabilidades incluidas dentro del modelo, estimadas para cada ciclo del modelo (semestral). Los años con calidad de vida fueron estimados como el producto del tiempo de permanecer en un estado por la medida de utilidad específica para cada estado que figura en la tabla 2.

\section{Costos}

Los costos del modelo fueron estimados empleando como referencia el precio máximo de regulación de las dosis promedio utilizadas para el manejo de la enfermedad (20 UI para detemir y glargina y una dosis promedio de 30 UI para insulina NPH), asumiendo que con estas dosis se mantiene al paciente en tratamiento durante los cinco años y que con ellas se obtiene un control de la enfermedad.

Se estimó una media ponderada de los costos de los eventos para el primer ciclo y para los ciclos posteriores, teniendo en cuenta eventos cardiovasculares (coronario agudo y cerebrovascular) y microvasculares (retinopatía y nefropatía). Lo anterior, a partir de una revisión de los registros del sistema de información de transacciones de diferentes entidades promotoras de salud (EPS), y tomando los valores reales pagados por una muestra de aseguradores a pesos colombianos del año 2014 (tabla 3).

\section{Análisis de sensibilidad}

Se realizó un análisis de sensibilidad multivariado tipo Montecarlo, utilizando como

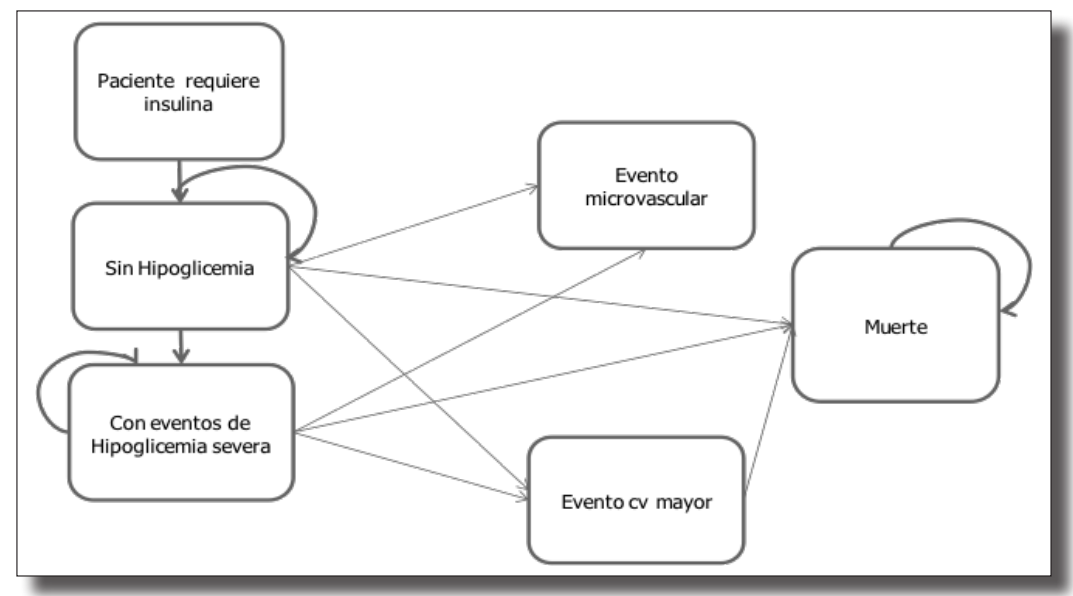

FIGURA 1. Modelo de Markov que Simula la historia NatURAl de la ENFERMEdad

Fuente: Romero et al. (12) 
TABLA 1. Probabilidad de PRESENTAR hipoglucemia gRaVe y PRobabilidad POR EVENTo

\begin{tabular}{|l|c|l|}
\hline Tecnología & Probabilidad & Estudio \\
\hline Detemir & 0,001093588 & Ross S, et al. $(17)$ \\
\hline Glargina & 0,080321285 & Ross S, et al. $(17)$ \\
\hline NPH & 0,1234569 & Rosenstock et al. $(16)$ \\
\hline Evento & $\begin{array}{c}\text { Paciente con antecedente de } \\
\text { hipoglucemia grave }\end{array}$ & Paciente sin antecedente de hipoglucemia grave \\
\hline $\begin{array}{l}\text { Evento macro- } \\
\text { vascular }\end{array}$ & {$[0,098-0,188]$} & {$[0,096-0,108](13)$} \\
\hline $\begin{array}{l}\text { Evento micro- } \\
\text { vascular }\end{array}$ & {$[0,065-0,143]$} & {$[0,096-0,107](13)$} \\
\hline Muerte & {$[0,144-0,256]$} & {$[0,053-0,156](13)$} \\
\hline
\end{tabular}

Fuente: elaboración propia basada en los datos obtenidos de los estudios referenciados

TABLA 2. UTILIDADES POR EVENTO

\begin{tabular}{|l|c|l|}
\hline Evento & Utilidades & Estudio \\
\cline { 1 - 2 } Eventos macrovasculares con hipoglucemia & 0,73 & \\
\cline { 1 - 2 } Eventos macrovasculares sin hipoglucemia & \multirow{2}{*}{ Solli (18) } & \\
\cline { 1 - 2 } Eventos microvasculares con hipoglucemia & 0,73 & \\
\cline { 1 - 2 } Eventos microvasculares sin hipoglucemia & 0,73 & Marrett (19) \\
\hline Hipoglucemia & $-0,205$ & Solli (18) \\
\hline Paciente sano & 0,81 & \\
\hline
\end{tabular}

Fuente: elaboración propia basada en los datos obtenidos de los estudios referenciados

rango los intervalos de confianza establecidos en el caso base, en cada ciclo, en un horizonte temporal de cinco años y desarrollando 1000 iteraciones para la cohorte de los 10000 pacientes. Teniendo en cuenta que fue utilizada una dosis promedio de 20 UI para detemir y glargina, y una dosis promedio de 30 UI para insulina nph, se desarrolló un análisis univariado igualando las dosis.

Como medida para evaluar la costo-efectividad se utilizó el umbral de tres PIB (producto interno bruto) per cápita para los AVAC, de acuerdo con lo propuesto por el IETS (14). De tal manera, tomando como referencia que el PIB per cápita reportado para Colombia en el año 2013 (15) fue de 7748 dólares, y que la tasa representativa del mercado (TRM) promedio fue de 1869,10 pesos colombianos por dolar para el año 2013 (15), el umbral para el análisis sería de 43445360,40 pesos por cada AVAC ganado. Además, con el propósito de estimar las razones de costo-efectividad, tanto a los costos como a los desenlaces se les aplicó una tasa de descuento de $5 \%$ anual (14).

\section{Resultados}

En el momento de la búsqueda no se encontraron estudios cabeza a cabeza de las tecnologías analizadas, pero el análisis permitió encontrar información más consistente 
TABLA 3. Costos EMPleados

\begin{tabular}{|l|c|l|}
\hline Variable & Costo & Fuente \\
\hline Paciente mes detemir & 52784 & Regulación \\
\hline Paciente mes glargina & 50150 & Regulación \\
\hline Paciente mes NPH & 9183 & Sismed \\
\hline Manejo y seguimiento detemir & 32967 & EPS, Sispro \\
\hline Manejo y seguimiento glargina & 32967 & EPS, Sispro \\
\hline Manejo y seguimiento NPH & $72658^{*}$ & EPS, Sispro \\
\hline Evento cardiovascular & 51324118,87 & RIPS-EPS \\
\hline Manejo en urgencias de un episodio de hipoglucemia & 771230 & RIPS \\
\hline Evento microvascular & 35926883,21 & RIPS-EPS \\
\hline
\end{tabular}

* Dentro del costo del manejo y seguimiento se incluyen los costos de aguja y jeringa que se necesitan para administrar la tecnología. Adicionalmente, la frecuencia en las consultas con especialista es mayor en esta tecnología que en las otras dos.

Fuente: elaboración propia

frente a la presentación de hipoglucemia (16-17) moderada y grave, la cual se ajustó en el modelo para realizar el análisis.

Los estudios seleccionados como insumo para obtener la utilidad que fue usada en el modelo fueron los de Solli et al. (18) y Marrett et al. (19). El primero evaluó la medida de utilidad de los pacientes que sufren complicaciones relacionadas con la diabetes, medida en la escala EQ-5D. Los autores encontraron que la enfermedad y sus complicaciones tienen un alto impacto en las dimensiones que evalúan la escala. En este estudio se puede observar que los eventos macrovasculares, accidente cerebrovascular, así como la neuropatía, son los que más afectan la medida de calidad de vida. Por otro parte, el estudio de Marret et al. muestra la asociación de los eventos de hipoglucemia con una disminución en la calidad de vida relacionada con la salud del paciente diabético tipo 2 .

Tras analizar el caso base, los resultados mostraron que los beneficios medidos en AVAC que se obtienen con el uso de detemir son mayores y su costo es menor, en com- paración con otras tecnologías analizadas. Con el uso de detemir el paciente que tiene DM2 obtiene una mayor efectividad, medida tanto en años de vida salvados (AVs) como en AVAC. El modelo muestra una disminución del número de eventos de hipoglucemia grave, los cuales llegan a ser solamente del 9,09\% del total de hipoglucemias con el uso de detemir, y asimismo un menor número de muertes y eventos micro y macrovasculares, en comparación con glargina e insulina NPH, las cuales están contempladas dentro de esta evaluación económica (tabla 4).

Los resultados en el número de eventos que se presentan con el uso de cada una de las moléculas hacen que los costos sean diferentes con el uso de cada tecnología. El costo con el uso de detemir es menor, con un valor de 11936084,53 pesos, debido a la menor tasa de presentación de eventos y su relación con los costos, mientras que para glargina e insulina NPH es de 12024805,20 pesos y 12011337,02 pesos, respectivamente. En relación con los AVAC y los AVs, detemir sigue siendo superior, con 2,604 para los primeros y 3,681 para los segundos, frente a glargina, 
con 2,545 y 3,641, e insulina NPH, con 2,533 y 3,632 , para AVAC y AVS, respectivamente.

En la tabla 5 se presentan los costos obtenidos del modelo, discriminados por el uso de cada tecnología, además del valor que acarrea el proceso de seguimiento y control. Se observa que detemir es la tecnología más costosa, pero tras evaluar el costo de los eventos que se presentan en los pacientes con las demás tecnologías, se obtiene un mayor costo asociado a estos eventos.

La estimación de la razón de costo-efectividad incremental (RCEI) de detemir, frente aglargina o insulina NPH, mostró que tanto desde el punto de vista de efectividad como desde el de la utilidad, esta sería dominante, debido a que se genera mayor efectividad y un menor costo (tabla 6). El ahorro generado cuando se usa detemir, frente aglargina, es de 88720 pesos, mientras que el ahorro frente a insulina NPH es de 75252 .

El resultado del análisis univariado que tenía en cuenta las dosis, encontró que detemir mantiene su dominancia frente a glargina, mientras que frente a insulina NPH pasa a ser costo-efectiva, y se encuentra por debajo del umbral de los tres PIB per cápita empleados en los análisis que se han realizado en el país.

El análisis de sensibilidad tipo Montecarlo, desarrollado para los dos escenarios (detemir frente a insulina NPH y detemir frente a glargina) arrojó como resultado que en la mayoría

TABLA 4. Resultados de datos globales de UnA COHORTE de 10000

\begin{tabular}{|l|l|c|c|c|c|}
\hline Tecnología & Costos & $\begin{array}{c}\text { Eventos de hipo- } \\
\text { glucemia grave }\end{array}$ & Muertes & $\begin{array}{c}\text { Eventos macro- } \\
\text { vasculares }\end{array}$ & $\begin{array}{c}\text { Eventos micro- } \\
\text { vasculares }\end{array}$ \\
\hline Detemir & 119360845284 & 10 & 865 & 842 & 835 \\
\hline Glargina & 120248051992 & 72 & 946 & 881 & 842 \\
\hline NPH & 120113370234 & 110 & 963 & 889 & 844 \\
\hline
\end{tabular}

Fuente: elaboración propia

TABLA 5. Resultados de Costo de un PACIENTE PROMEdio discriminado POR CATEgoría

\begin{tabular}{|l|c|c|c|}
\hline Categoría & Detemir & Glargina & NPH \\
\hline Tecnología y costos de seguimiento y control & 4615539,42 & 4473674,19 & 4404912,21 \\
\hline Hipoglucemia & 756,61 & $5.534,73$ & $8.488,89$ \\
\hline Eventos macrovasculares & 4319134,56 & 4520390,09 & 4564441,29 \\
\hline Eventos microvasculares & 3000653,94 & 3025206,19 & 3033494,63 \\
\hline Total & 11936084,53 & 12024805,20 & 12011337,02 \\
\hline
\end{tabular}

Fuente: elaboración propia

TABLA 6. Resultado de RAZONES de COSTO EFECTIVIDAd PARA UN PACIENTE EN PROMEDIO

\begin{tabular}{|l|c|c|c|c||c|}
\hline \multirow{2}{*}{ Escenario } & \multicolumn{3}{|c|}{ Incrementales } & \multirow{2}{*}{ RCEI (AVs) } & \multirow{2}{*}{ RCUI (AVAC) } \\
\cline { 2 - 5 } & AVS & AVAC & Costo & & \\
\hline Detemir $v s$. glargina & 0,040 & 0,059 & $(88720,67)$ & Dominante & Dominante \\
\hline Detemir $v s . \mathrm{NPH}$ & 0,049 & 0,071 & $(75252,50)$ & Dominante & Dominante \\
\hline
\end{tabular}

Fuente: elaboración propia 
TABLA 7. ANÁLISIS dE SENSIBILIDAD UNIVARIAdo

\begin{tabular}{|l|c|c|}
\hline Escenario & RCEI (AVs) & RCUI (AVAC) \\
\hline $\begin{array}{l}\text { Detemir } v s . \text { glar- } \\
\text { gina }\end{array}$ & Dominante & Dominante \\
\hline Detemir $v s . \mathrm{NPH}$ & 27533127,05 & 18805100,86 \\
\hline
\end{tabular}

Fuente: elaboración propia

de las iteraciones detemir se encuentra en el plano de dominancia. Este análisis se realizó con el fin de minimizar la incertidumbre de los resultados obtenidos si se presentara un cambio en alguna de las variables del modelo, en más o menos el $20 \%$ (figuras 2 y 3 ).

\section{Discusión}

Esta nueva evaluación económica compara detemir con glargina e insulina $\mathrm{NPH}, \mathrm{y}$ muestra, posteriormente a los ajustes de los nuevos precios de los medicamentos en Colombia y la actualización de la información científica disponible hasta el año 2014, los resultados actualizados y la inclusión del desenlace de calidad de vida.

Los resultados de esta evaluación dejan ver cómo el uso de detemir sería, desde las condiciones expuestas para el presente análisis, la opción del mejor resultado en efectividad y costo, en un horizonte temporal de cinco años, manteniendo la tendencia presentada en la evaluación que desarrollaron Romero et al. (12), pero ahora en dominancia por el factor costo del medicamento, ya que este disminuyó en más del $30 \%$ debido a la regulación de medicamentos realizada en el 2014 por el Ministerio de Salud colombiano.

La diferencia fundamental encontrada entre estas tecnologías se basa en la probabilidad de que se presenten eventos de hipoglucemia moderados y/o graves, $y$, en consecuencia, como lo han publicado diferentes estudios de seguimiento de población, en el riesgo de eventos macro y microvasculares ocasionados por la presencia de condiciones de hipoglucemia. Aunque es importante reconocer que aún no se determina la causa de esta asociación, estudios como el de Zoungas et al. mantienen esa relación (13). Esto muestra la necesidad de desarrollar nuevos estudios regionales que refrenden estos resultados.

Esta diferencia, en la que detemir muestra superioridad frente a insulina NPH y glargina, hace que en el desenlace final de muertes evitadas y de Avs se generen mejores resultados que con las otras tecnologías y, simultáneamente, que con los nuevos costos para Colombia se presenten resultados de ahorro. Esto concuerda con el estudio de Home et al. (20), quienes evaluaron la costo-efectividad mediante los AVAC en cinco países, en pacientes que inician tratamiento con insulina luego de un inadecuado control con el uso de hipoglucemiantes orales. Los resultados fueron presentados como fracción del PIB per cápita de cada país, y como rentabilidad se definía un RCEI <3,0. En todos los países evaluados se encontró por debajo de este límite, lo cual demuestra sus ventajas como intervención costo-efectiva en diferentes tipos de sistemas de salud.

Por su parte, en el estudio de Ridderstråle et al. (21) se encontró que detemir, comparada con insulina NHP, disminuye el riesgo de hipoglucemia grave, y de esa manera se mejora la calidad de vida de los pacientes, hecho que se ve reflejado en los resultados del modelo analizado en este trabajo.

Aunque en el país no existen estudios similares que evalúen el uso de estas tres moléculas, al comparar los resultados de este análisis con los del estudio de Ridderstråle et al. (realizado en Finlandia, Noruega y Suecia) (21), se puede evidenciar que son similares, debido a que comparan detemir frente a NPH y señalan que el bajo riesgo de presentación 
ANÁLISIS DE COSTO-UTILIDAD DEL USO DE DETEMIR EN PACIENTES CON DIABETES TIPO 2

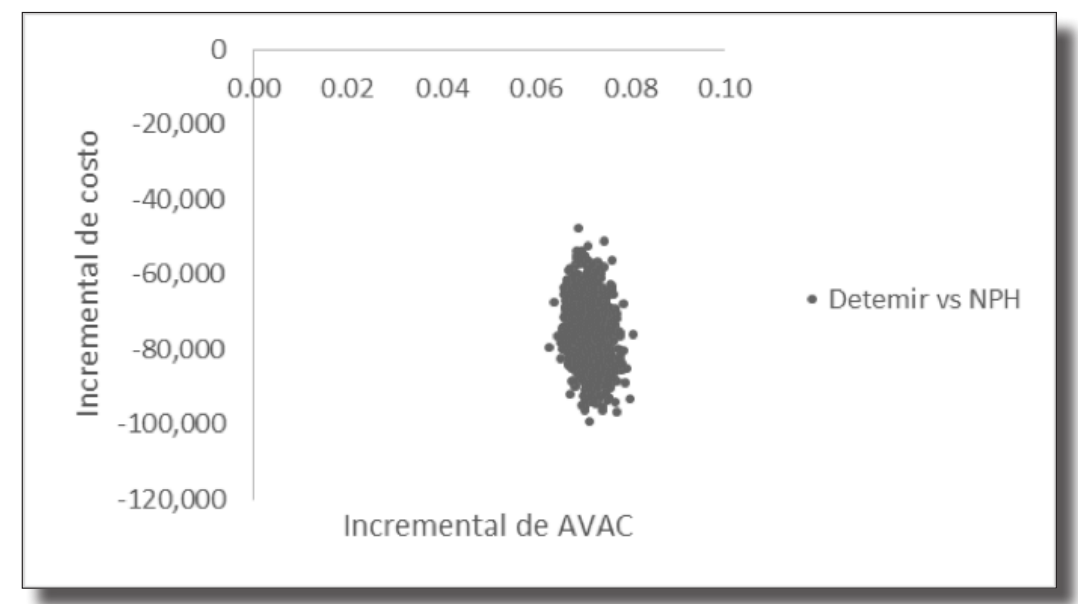

FIGURA 2. ANÁLISIS DE SENSIBILIDAD DETEMIR VERSUS NPH

Fuente: elaboración propia

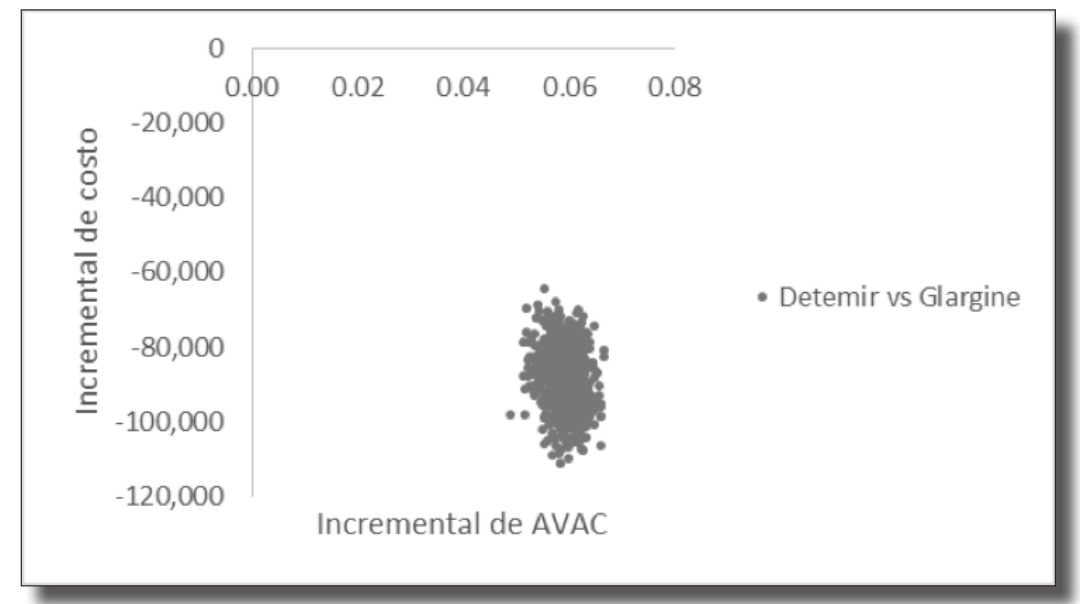

FIGURA 3. ANÁLISIS DE SENSIBILIDAD DETEMIR VERSUS GLARGiNa

Fuente: elaboración propia

de hipoglucemias no graves asociadas al uso de detemir, hacen que esta sea una opción que proporciona beneficios económicos; esta conclusión valida los resultados obtenidos en el presente estudio.

Por otra parte, el estudio realizado por Tunis et al. (22), que evaluó los AVAC en pacientes con diabetes tipo 2 en Canadá, encontró que el incremental ganado con el uso de detemir es de 0,475 años, frente a 0,305 con el uso de insulina NPH. Los resultados, al igual que en este estudio, se asocian a los menores costos de las complicaciones, debido a la diferencia en la tasa de presentación de eventos de hipoglucemia y la poca desutilidad que generan.

Teniendo en cuenta que las tres opciones terapéuticas están incluidas dentro del plan de beneficios colombiano (POS), el resultado de la evaluación muestra cuál sería el ahorro final que podría significar para el sistema de salud el uso de una u otra tecnología, además 
de la protección frente a los desenlaces finales de la enfermedad, ante la no presentación de eventos macro o microvasculares.

La complejidad misma de la enfermedad, y de los efectos de los tratamientos, hace que ciertos elementos, como la disminución del peso y de la medida de la cintura abdominal, que pueden reflejarse como un menor riesgo cardiovascular y que también son efectos de detemir, no queden plasmados en este tipo de evaluaciones, pero que podrían aumentar el beneficio de su uso, tanto en resultados finales como en calidad de vida.

Dentro de las limitaciones de este estudio se considera que no fue posible tomar información del país en términos de calidad de vida. Los resultados encontrados, donde se publican de manera detallada las utilidades y desutilidades de pacientes con diabetes mellitus y la presentación de eventos de hipoglucemia, hicieron posible establecer una medida, y así estimar de manera consolidada los resultados, además de mostrar la mayor efectividad de detemir en términos de años con calidad de vida. Otra de las limitaciones de este análisis, y la más relevante, es la incertidumbre que se genera frente a lo que podría ocurrir en la población colombiana, aunque los beneficios se sustentan en estudios multicéntricos y el modelo es robusto por su componente probabilístico, la ausencia de datos de la población colombiana limita los resultados.

Por otra parte, se debe pensar en realizar estudios sobre los reales efectos de los eventos asociados con la diabetes tipo 2 en pacientes del país, principalmente si se tiene en cuenta que las tecnologías en general son de amplio uso en Colombia, en especial cuando el factor fundamental de desenlace se basa en las diferencias frente a la presentación de hipoglucemias.

\section{Conclusión}

En este estudio se puede ver cómo la efectividad de detemir en pacientes con diabetes mellitus genera beneficios en AVS y AVAC, lo cual hace que esta opción deba ser tenida en cuenta para el manejo de pacientes que padecen DM2. Adicionalmente, se pueden generar ahorros dentro del sistema de salud por la adopción de esta tecnología, debido a que dentro del estudio solo se utilizó el precio de regulación, entendido como el precio máximo de esta tecnología, por lo que se podría generar un ahorro mayor al tomar un costo de transacción que por las leyes del mercado tendería a ser más bajo que su precio de regulación.

De acuerdo con los resultados encontrados en la evaluación de costo-efectividad y costoutilidad realizada en el presente estudio, se puede concluir que detemir sería la opción más favorable a tener en cuenta para el manejo de pacientes con DM2 que no han controlado la enfermedad con la farmacoterapia oral, por su efectividad y costos dentro del sistema de salud.

\section{Referencias bibliográficas}

1. International Diabetes Federation (IDF). Diebetes Atlas. Sexta edición [Internet]. Bruselas: IDF; 2014. Disponible en: www.idf.org/diabetesatlas.

2. American Diabetes Association (ADA). Standards of Medical Care in Diabetes-2015. Diabetes Care. 2015; 38 (1): S1-S94.

3. Federación Internacional de Diabetes. Atlas de la diabetes de la FID [Internet], 2014 [acceso: 30 de abril del 2015]. Disponible en: http://www.idf.org/sites/ default/files/Atlas-poster-2014_ES.pdf.

4. Asociación Latinoamericana de Diabetes (ALAD). Guias ALAD sobre diagnóstico, control y tratatmiento de la diabetes mellitus tipo 2 con medicina basada en la evidencia 2013. Revista de la ALAD [Internet]. 2014. Disponible en: http://issuu.com/alad-diabetes/docs/ guias_alad_2013.

5. Ampudia-Blasco FJ, Rosenstock J. Estrategias de insulinización en la diabetes mellitus tipo 2. Av Diabetol. 2008; 24 (1): 7-20.

6. Singh SR, Ahmad F, Lal A, Yu C, Bai Z, Bennett H. Efficacy and Safety of Insulin Analogues for the 
Management of Diabetes Mellitus: A Meta-Analysis. CMAJ. 2009; 180 (4): 385-97.

7. Pieber TR, Treichel HC, Hompesch B, Philotheou A, Mordhorst L, Gall MA, et al. Comparison of Insulin Detemir and Insulin Glargine in Subjects with Type 1 Diabetes Using Intensive Insulin Therapy. Diabet Med. 2007; 24 (6): 635-42. Epub 2007; Mar 22.

8. Zilova A, Naggar N, Shahc S, Shend C, Haddade J. Insulin Detemir in the Management of Type 2 Diabetes in Non-Western Countries: Safety and Effectiveness Data from the Achieve Observational Study. Diabetes Research and Clinical Practice. 2013; 101: 317-25.

9. Göz F, Karaoz S, Goz M, Ekiz S, Cetin I. Effects of the Diabetic Patients Perceived Social Support on their Quality-of-Life. J Clin Nurs. 2007; 16 (7): 1353-60.

10. Rubin R, Peyrot M. Quality of Life and Diabetes. Diabetes/Metabolism Research and Reviews. 1999 ; 15 (3): 205-18. doi: 10.1002/(SICI) 1520 7560(199905/06)15:3<205::AID-DMRR29>3.0.CO;2-O.

11. Huang ES, Brown SE, Ewigman BG, Foley EC, Meltzer DO. Patient Perceptions of Quality of Life with Diabetes-Related Complications and Treatments. Diabetes Care. 2007; 30 (10): 2478-83. Epub 2007; Jul 10.

12. Romero M, Alvis-Guzmán N, Chávez D, Karpf E. Análisis de costo-efectividad del uso de Detemir en pacientes con diabetes tipo 2 en riesgo de presentar eventos cardiovasculares y muerte en Colombia. Revista Científica Salud Uninorte [Internet]. 2014; 30 (2): 200-209. Disponible en: http://rcientificas.uninorte. edu.co/index.php/salud/article/view/4720/6314.

13. Zoungas S, Patel A, Chalmers J, de Galan B, Li Q, Billot L. et al. Severe Hypoglycemia and Risks of Vascular Events and Death. N Engl J Med. 2010; 363 : 1410-8. doi: 10.1056/NEJMoa1003795.

14. Instituto de Evaluación Tecnológica en Salud (IETS). Manual para la elaboración de evaluaciones económicas en salud [Internet]. Bogotá: IETS; 2014. Disponible en: http://www.iets.org.co/Manuales/ Manuales/Manual\%20evaluacio $\%$ CC $\% 81$ n $\% 20$ econo $\%$ CC $\% 81$ mica $\% 20$ web $\% 2030 \% 20$ sep.p.

15. Banco de la República de Colombia. Series estadísticas. Tasa de cambio. Bogotá: Banco de la República [Internet]; 2013. Disponible en: http://www.banrep. gov.co/series-estadisticas/see_ts_cam.htm.
16. Rosenstock J, Fonseca V, Schinzel S, Dain MP, Mullins P, Riddle M. Reduced Risk of Hypoglycemia with Once-Daily Glargine versus Twice-Daily NPH and Number Needed to Harm with NPH to Demonstrate the Risk of One Additional Hypoglycemic Event in Type 2 Diabetes: Evidence from a Long-Term Controlled Trial. J Diabetes Complications. 2014; 28 (5): 742-9. doi: 10.1016/j.jdiacomp.2014.04.003. Epub 2014 Apr 16.

17. Ross S, Dzida G, Ji Q, Kaiser M, Ligthelm R, Meneghini L, et al. Safety of Once-Daily Insulin Detemir in Patients with Type 2 Diabetes Treated with Oral Hypoglycemic Agents in Routine Clinical. J Diabetes. 2014; 6 (3): 243-50. doi: 10.1111/17530407.12091. Epub 2013 Oct 29.

18. Solli O, Stavem K, Kristiansen IS. Health-Related Quality of Life in Diabetes: The Associations of Complications with EQ-5D Scores. Health and Quality of Life Outcomes. 2010; 8 (1): 18. doi: 10.1186/1477-7525-8-18.

19. Marrett E, Radican L, Davies MJ, Zhang Q. Assessment of Severity and Frequency of SelfReported Hypoglycemia on Quality of Life in Patients with Type 2 Diabetes Treated with Oral Antihyperglycemic Agents: A Survey Study. BMC Res Notes. 2011; 4: 251. doi: 10.1186/1756-0500-4-251.

20. Home P, Hyun S, González G, Malek R, Nikolajsen A. An Analysis of the Cost-Effectiveness of Starting Insulin Detemir in Insulin-Naïve People with Type 2 Diabetes. J Med Econ. 2014; 21. doi:10.3111/136 96998.2014.985788.

21. Ridderstråle M, Jensen MM, Gjesing RP, Niskanen L. Cost-Effectiveness of Insulin Detemir Compared with NPH Insulin in People with Type 2 Diabetes in Denmark, Finland, Norway, and Sweden. J Med Econ. 2013; 16 (4): 468-78. doi: 10.3111/13696998.2013.768999. Epub 2013 Feb 6.

22. Tunis SL, Minshall ME, Conner C, McCormick JI, Kapor J, Yale JF, et al. Cost-effectiveness of Insulin Detemir Compared to NPH Insulin for Type 1 and Type 2 Diabetes Mellitus in the Canadian Payer Setting: Modeling Analysis. Current Medical Research and Opinion. 2009; 5 (25): 1273-84. 